\title{
Optimization of the COD Removal Efficiency for a Static Granular Bed Reactor Treating Poultry Slaughterhouse Wastewater
}

\author{
Z Rinquest*, M Basitere**, M Mewa-Ngongang*, SKO Ntwampe*, M Njoya* \\ * Bioresource Engineering Research Group (BioERG), Department of Biotechnology, \\ Cape Peninsula University of Technology, P. O. Box 652, Cape Town 8000, South Africa \\ (E-mail: zainab.rinquest@gmail.com; mewamaxwell@gmail.com; ntwampeS@cput.ac.za; \\ mahomet.njoya@hotmail.com) \\ ** Department of Chemical Engineering, Cape Peninsula University of Technology, P. O. \\ Box 652, Cape Town 8000, South Africa, (E-mail: BasitereM@cput.ac.za)
}

\begin{abstract}
In this study, the efficiency of an anaerobic treatment system for wastewater from a South African poultry slaughterhouse was evaluated using a lab-scale static granular bed reactor (SGBR). The down-flow SGBR $(2 \mathrm{~L})$ was operated continuously for 138 days under mesophilic conditions $\left(35-37^{\circ} \mathrm{C}\right)$, at hydraulic retention times (HRTs) ranging from 24 to $96 \mathrm{~h}$ and average organic loading rates (OLRs) of 0.78 to $5.74 \mathrm{~g} \mathrm{COD/Lday.} \mathrm{The} \mathrm{SGBR} \mathrm{achieved} \mathrm{an} \mathrm{average} \mathrm{chemical}$ oxygen demand (COD) removal efficiency of $80 \%$ and the maximum COD removal achieved was 95\%, at an HRT of $24 \mathrm{~h}$ and average OLR of $5.74 \mathrm{~g}$ COD/Lday. The optimization of the SGBR, with regard to a suitable HRT and OLR, was determined using response surface methodology (RSM). The optimal SGBR performance with regard to the maximum COD removal efficiency was predicted for an OLR of $12.49 \mathrm{~g}$ COD/Lday and a HRT of $24 \mathrm{~h}$, resulting in a $95.5 \%$ COD removal efficiency. The model R2 of 0.9638 indicated that the model is a good fit and is suitable to predict the COD removal efficiency for the SGBR.
\end{abstract}

\section{Keywords}

Anaerobic digestion; Chemical oxygen demand; Optimization; Poultry slaughterhouse wastewater; Response surface methodology; Static Granular Bed Reactor

\section{INTRODUCTION}

The SGBR is a relatively new high-rate anaerobic reactor developed with the purpose of simplifying the design, operation, and maintenance of high-rate anaerobic reactors whilst still maintaining high-quality influent treatment and maximizing biogas production (Ellis, 2008; Evans, 2004). The design of the SGBR is based on the up-flow anaerobic sludge blanket (UASB) reactor design; however, the distinguishing feature of the SGBR is its down-flow configuration which eases the separation of the wastewater, solids, and biogas. Unlike other down-flow anaerobic reactors, such as the anaerobic filter (AF), the SGBR uses highly active anaerobic granules (Debik \& Coskun, 2009; Evans, 2004). In addition, the down-flow configuration of the SGBR enables the accommodation of higher suspended solid concentrations in comparison to up-flow anaerobic reactors, such as the UASB reactor and expanded granular sludge bed (EGSB) reactor, which 
frequently experience solids washout, i.e. the loss of granular biomass due to the high up-flow velocities used. There are bioreactor conditions which must be monitored and maintained to ensure that anaerobic and aerobic bioreactors operate optimally and more importantly to avoid process inhibition and system failure. Firstly, the micro-organisms used must have sufficient time for the biodegradation of organic matter and the sorption of unreactive species, and secondly, the organic loading must be controlled in such a manner that overfeeding of the micro-organisms is prevented (Nayona, 2010). The HRT and OLR are two of the key operating conditions used to ensure that an appropriate balance is achieved in bioreactors treating PSW (Wellinger et al., 2013). These conditions can be optimized using statistical methods, such as RSM.

The SGBR has been extensively used for the treatment of wastewaters from different industries, including poultry slaughterhouse wastewater (PSW) (Chong et al., 2012; Park et al., 2012). According to the literature reviewed, mainly laboratory and pilot-scale SGBRs operating at ambient temperature (i.e. approximately $25^{\circ} \mathrm{C}$ ), have been researched. The pilot-scale SGBR systems are being used for wastewater treatment with the intention of studying the effect of the HRT, solids retention time (SRT), and OLR. The data collected from such pilot-scale studies are to be used for the design of full-scale SGBR systems and the commercialization of this new technology (Park et al., 2012). Variations of the SGBR operating conditions, i.e. temperature, OLR and HRT, have proven to have relatively minimal effect on the stability of the SGBR and do not significantly affect the effluent quality (Ellis, 2008). Therefore, the aim of this study was to: 1) determine the feasibility of treating PSW using a SGBR and 2) optimize the SGBR with regard to the HRTs and OLRs using RSM.

\section{MATERIALS AND METHODS}

\section{PSW characterization, SGBR setup and inoculation}

PSW was sourced from a poultry product processing plant located in the Western Cape Province (South Africa). The fresh PSW samples were stored in $25 \mathrm{~L}$ polyvinyl chloride (PVC) containers and were refrigerated at $4{ }^{\circ} \mathrm{C}$. The characteristics of the PSW used in this study, specifications of the PSW and anaerobic granules used for the SGBR inoculation are listed in Table 1. A lab-scale down-flow SGBR, made from a cylindrical glass column, with an active volume of $2 \mathrm{~L}$ (height and inner diameter of $0.62 \mathrm{~m}$ and $0.065 \mathrm{~m}$, respectively), was used in this study. A perforated influent tube was positioned at the top of the SGBR to evenly distribute the influent. A second perforated tube was positioned in the centre of the underdrain bed as part of a backwashing system, which also had an overflow line. Pumice stones (average diameter of 5-20 $\mathrm{mm}$ ) were used as the underdrain bed to prevent the washout of the anaerobic granules. A stainless-steel sieve (pore size: $2 \mathrm{~mm}$ ) was fixed to the bottom of the SGBR to retain the pumice stones, which occupied a volume of $0.3 \mathrm{~L}$. The SGBR system consisted of a $5 \mathrm{~L}$ feed storage tank, a $5 \mathrm{~L}$ effluent storage tank, polyvinyl chloride (PVC) and glass fittings, silicone tubing, a MINIPULS® Evolution peristaltic pump (Gilson, USA), and a circulating water bath were used to supply warm water to the jacket (Figure 1). The SGBR was inoculated with $0.4 \mathrm{~L}$ anaerobic granules obtained from a full-scale mesophilic up-flow anaerobic sludge blanket (UASB) reactor operated at a local brewery plant (Newlands, South Africa) and fed with 1.6 L of PSW. A dry milk solution of $0.01 \mathrm{~L}(1427 \pm 65 \mathrm{mg}$ COD/L) prepared with potable water $(50 \%(\mathrm{v} / \mathrm{v}))$ was used to facilitate the acclimatization of the anaerobic granules. The specifications of the anaerobic granules and PSW used are presented in Table 1B and C. The SGBR feed was prepared by filtering the untreated PSW through a mesh screen (pore size: 2 $\mathrm{mm}$ ) to remove floating solids, which could potentially clog tubing and cause operational challenges. The filtered PSW was then diluted $(50 \%$ and $25 \%(\mathrm{v} / \mathrm{v}))$ with potable water to prevent shock loading of the SGBR during the start-up period. After the acclimatization period $(48 \mathrm{~h})$, the HRT was set to 2.3 days $(55 \mathrm{~h})$ to initiate the start-up OLR of $1.18 \mathrm{~g} \mathrm{COD/L.day.} \mathrm{A} \mathrm{start-up} \mathrm{OLR} \mathrm{of}$ approximately $1 \mathrm{~g} \mathrm{COD/L.day} \mathrm{is} \mathrm{recommended} \mathrm{for} \mathrm{SGBRs} \mathrm{based} \mathrm{on} \mathrm{previous} \mathrm{studies} \mathrm{(Park} \mathrm{et} \mathrm{al.,}$ 2012; Lim, 2008). The HRT was maintained at $55 \mathrm{~h}$ and the SGBR was operated in a continuous 
mode under steady state conditions, throughout the start-up period (28 days). As the performance of the SGBR stabilized with regard to the percentage COD removal, the HRT was varied stepwise to simulate variations in the feed flow rate and thus the OLR so as to adequately assess the performance of the SGBR in treating PSW. The SGBR was maintained at mesophilic temperature (i.e. $35-37^{\circ} \mathrm{C}$ ) throughout the 138 days of continuous, steady-state operation.

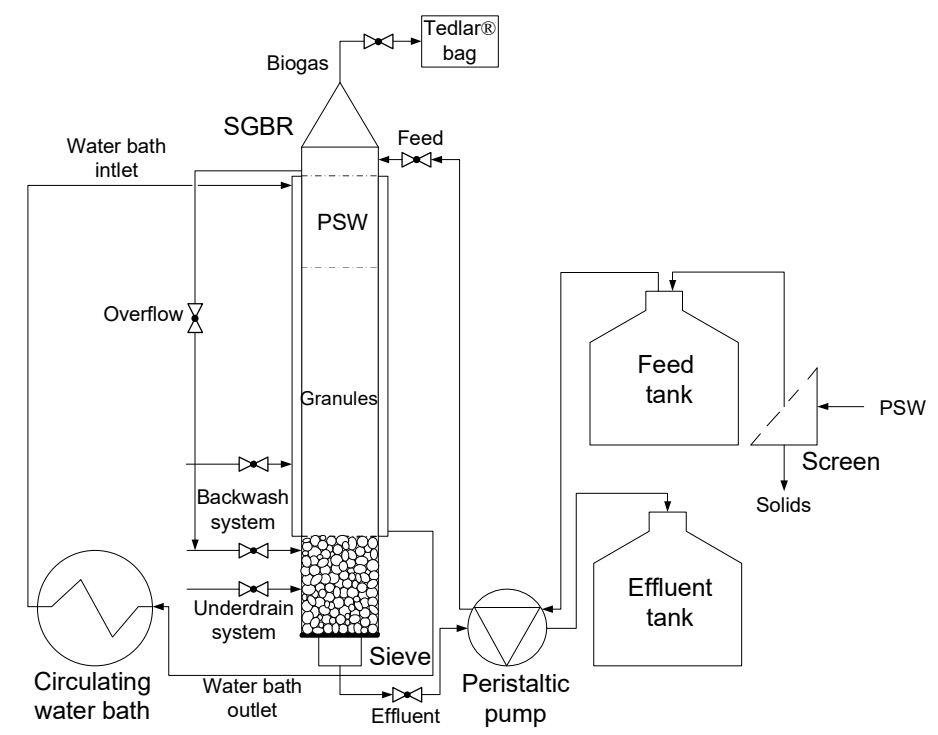

Figure 1 Schematic diagram of the lab-scale SGBR system

Table 1 A) Characteristics of the untreated PSW used in this study, B) Specifications of the PSW and $C$ ) anaerobic granules used for the SGBR inoculation

\begin{tabular}{llll}
\hline & $\mathrm{A}$ & $\mathrm{B}$ & $\mathrm{C}$ \\
\cline { 2 - 4 } Parameter (Unit) & Values & Values & Values \\
\hline Chemical oxygen demand (COD) mg/L & 5216 & $2800 \pm 36$ & - \\
$\mathrm{pH}$ at $25^{\circ} \mathrm{C}$ & 5.6 & 7.5 & 7.5 \\
Conductivity at $25{ }^{\circ} \mathrm{C} \mathrm{mS} / \mathrm{m}$ & 159 & - & - \\
Ammonia (as N) $\mathrm{mg} / \mathrm{L}$ & 25.2 & 215 & - \\
Ortho-phosphate $\left(\mathrm{PO}_{4}{ }^{3-}\right) \mathrm{mg} / \mathrm{L}$ & 24.3 & 31 & - \\
Nitrate $\left(\mathrm{NO}_{3}{ }^{-}\right.$) $\mathrm{mg} / \mathrm{L}$ & 1.0 & 1.9 & - \\
Total suspended solids (TSS) mg/L & 1580 & 675 & 42867 \\
Volatile suspended solids (VSS) mg/L & 1533 & 1625 & 51391 \\
Volatile fatty acids (VFA) mg/L & 421 & - & - \\
\hline
\end{tabular}

Table 2 Summary of SGBR operating conditions

\begin{tabular}{lllll}
\hline $\begin{array}{l}\text { Operating time } \\
\text { (days) }\end{array}$ & $\begin{array}{l}\text { HRT } \\
(\mathrm{h})\end{array}$ & $\begin{array}{l}\text { Flow rate }(\mathrm{Q}) \\
(\mathrm{L} / \mathrm{h})\end{array}$ & $\begin{array}{l}\text { Average OLR } \\
(\mathrm{g} \text { COD/Lday) }\end{array}$ & $\begin{array}{l}\text { Dilution (v/v) (\%) } \\
\text { [number of days] }\end{array}$ \\
\hline 28 & 55 & 0.0364 & 1.18 & $50 \quad[28]$ \\
29 & 96 & 0.0208 & 0.78 & $50 \quad[15]$ \\
& & & & $75 \quad[8]$ \\
27 & & & & None [6] \\
28 & 48 & 0.0364 & 1.96 & None \\
26 & 36 & 0.0486 & 4.10 & None \\
\hline
\end{tabular}




\section{Response surface methodology}

RSM was used for the optimization of the COD removal efficiency for the SGBR treating PSW. A central composite design (CCD) was used to determine the optimum operating conditions, i.e. hydraulic retention time (HRT) (B) and organic loading rate (OLR) (A), and the interaction between these independent variables, with the COD removal efficiency (\%) being the response variable. The OLR and HRT were chosen based on their direct impact on the COD removal efficiency and the chosen ranges were based on the operating conditions used for the SGBR in this study, i.e. conditions reported in Debik \& Coskun (2009) and Basitere et al. (2017). Design Expert ${ }^{\circledR} 10.0 .3$ statistical software (Stat-Ease, Inc., USA) was used for the experimental design. A two-factor (i.e. HRT and OLR), two-level (i.e. low (-1) and high (+1)) CCD was applied, with a total of 15 experimental runs generated. This corresponded to $0.73(\min )$ to $12.5(\max )$ and $1(\min )$ to $4(\max )$ for OLRs and HRTs. Analysis of variance (ANOVA) was used to evaluate the significance of the response surface quadratic model, the individual variables and their interactions (Bustillo-Lecompte \& Mehrvar, 2017).

\section{Sampling and analysis}

Feed and effluent samples were collected at $24 \mathrm{~h}$ intervals (i.e. Mondays, Wednesdays, and Fridays) and analysed in triplicate. The COD analysis samples were prepared using Merck COD solutions (solution A: Cat. No. 1.14538.0065 and 1.14679.049; solution B: Cat. No. 1.14539.0495 and 1.14680.1495), digested in a preheated Spectroquant TR420 Thermoreactor, and measured using the Merck Spectroquant Nova60. A weekly composite sample of the feed and effluent were sent to a South African National Accreditation System (SANAS) accredited lab (Scientific Services, South Africa), for confirmation of the COD results.

\section{RESULTS AND DISCUSSION SGBR performance: COD Removal}

During the 138 days of operation, the COD removal efficiencies exceeded $80 \%$, with the intermittent performance reduction on several days being attributed to backwashing operations. Similarly, the low initial COD removal efficiencies (average $74.1 \pm 3.6 \%$ ) observed during the startup period could be as a result of reduced FOG hydrolysis and suspended solids interaction with the anaerobic granules which contributed to an increase in soluble COD non-biodegradation. Del Nery et al. (2007) reported 65\% COD and $85 \%$ soluble COD removal efficiencies for the treatment of PSW in a UASB at an average OLR of $1.64 \mathrm{~g}$ COD/L.day. The average COD removal efficiencies of $78 \pm 4 \%, 79 \pm 5 \%, 86 \pm 5 \%$ and $85 \pm 5 \%$, for the corresponding HRTs of 96, 48, 36 and $24 \mathrm{~h}$, respectively, was attributed to the increased functionality of the biomass with increasing bioreactor operation time, thus the SGBRs ability to effectively tolerate increasing OLRs as the biomass matured. Several studies using the SGBR to treat a variety of wastewaters showed a similar trend (Debik \& Coskun, 2009; Lim \& Fox, 2011; Park et al., 2012; Oh et al., 2015). Despite fluctuations in the COD of the feed, an OLR of up to $12.5 \mathrm{~g} \mathrm{COD/Lday,} \mathrm{and} \mathrm{step-wise} \mathrm{decreases} \mathrm{in} \mathrm{the} \mathrm{HRT,}$ culminated in the COD removal efficiencies remaining relatively consistent. Overall, the SGBR design used in this study demonstrated a stable performance with COD removal efficiencies greater than $95 \%$ observed towards the end of the study at the $24 \mathrm{~h}$ HRT. Comparatively, the SGBR is known to produce COD removal efficiencies exceeding 90\% for a wide range of HRTs, i.e. 9 to 55 $\mathrm{h}$ and OLRs i.e. 0.63 to $9.72 \mathrm{~g} \mathrm{COD/Lday,} \mathrm{under} \mathrm{varying} \mathrm{temperature} \mathrm{conditions} \mathrm{(Debik} \mathrm{\&} \mathrm{Coskun,}$ 2009; Lim \& Fox, 2011; Park et al., 2012; Oh et al., 2015; Basitere et al., 2017), which is indicative of the robustness of the SGBR. Whilst it is evident that the HRT and OLR affects the COD removal efficiency, in this study neither an increase nor decrease in the HRT or OLR had long lasting impact nor adverse effects on the overall biological performance of the SGBR in terms of its COD removal efficiency. This indicated that the high biomass concentration in the SGBR was sufficient to maintain the required high removal efficiencies for a wide range of HRTs and OLRs. Debik \& 
Coskun (2009) operated a SGBR at HRTs of 48 and $36 \mathrm{~h}$ for the treatment of PSW; and achieved COD removal efficiencies higher than 90\% at OLRs ranging between 2.53 and $4.97 \mathrm{~g} \mathrm{COD} / \mathrm{Lday}$. Similarly, Basitere et al. (2017) reported COD, TSS and FOG removal efficiencies of 93\%, 95\% and $90 \%$, respectively, when operating a SGBR for PSW treatment at HRTs of 55 and $40 \mathrm{~h}$ with average OLRs of 1.01 and $3.14 \mathrm{~g} \mathrm{COD/Lday.}$

\section{Optimization of the COD removal efficiency for the SGBR}

Response surface methodology (RSM) was used for the optimization of the SGBR operating conditions through the development of a quadratic model used to predict the COD removal efficiency of the SGBR for the treatment of PSW. The hydraulic retention time (HRT) and organic loading rate (OLR) were selected as the two independent variables which were evaluated in order to determine their effect on the COD removal efficiency. SGBRs treating slaughterhouse wastewaters are capable of operating under HRTs ranging from 8 to $96 \mathrm{~h}$ (0.33 to 4 days) (Oh, 2012; Mach, 2004) and OLRs as high as $12.76 \mathrm{~g} \mathrm{COD/Lday} \mathrm{(Park} \mathrm{et} \mathrm{al.,} \mathrm{2012).} \mathrm{The} \mathrm{chosen} \mathrm{ranges} \mathrm{used} \mathrm{were}$ based on the operating conditions used for the SGBR in this study, i.e. conditions reported in Debik \& Coskun (2009) and Basitere et al. (2017). The SGBR system used in this study was able to consistently reduce the organic matter content of the PSW, resulting in an overall COD removal efficiency of $80 \%$ for HRTs of between 1 to 4 days $(24 \mathrm{~h}$ to $96 \mathrm{~h})$ and an average OLR of $2.75 \mathrm{~g}$ COD/L.day. Table 3 shows the central composite design (CCD) of the independent variables used to optimize the COD removal efficiency. The predicted results from the CCD indicated that an OLR of $12.49 \mathrm{~g} \mathrm{COD/Lday} \mathrm{(A)} \mathrm{and} \mathrm{a} \mathrm{HRT} \mathrm{(B)} \mathrm{of} 1$ day were the optimum conditions for attaining the maximum COD removal efficiency $(95.5 \%)$ for the SGBR used for the treatment of the PSW. The overall results suggest that the COD removal efficiency attained by the SGBR increased as the organic strength of the PSW increased and the HRT was decreased. Conversely, Basitere et al. (2017) reported a decrease in the COD removal efficiencies of an SGBR treating PSW when the OLR increased subsequent to a decrease in the HRT from $55 \mathrm{~h}$ to $48 \mathrm{~h}$. The interaction between the operating conditions, i.e. HRT and OLR, and the COD removal efficiency was determined using a polynomial regression. Table 4 summarizes the analysis of variance (ANOVA) results for the quadratic model used to predict the COD removal efficiency of the SGBR. Eq. 2 represents the resultant quadratic model which best fit the regression results.

Table 3 CCD of the independent variables, (A) OLR and (B) HRT

\begin{tabular}{lllll}
\hline & Factors & \multicolumn{3}{c}{ COD Removal efficiency (\%) } \\
\cline { 2 - 5 } 1 & OLR (A) (g COD/Lday) & HRT (B) (day) & Experimental & Predicted \\
2 & 1.04 & 2.29 & 72.8 & 72.4 \\
3 & 0.96 & 2.29 & 67.7 & 71.7 \\
4 & 1.26 & 2.29 & 76.0 & 74.1 \\
5 & 0.73 & 4 & 78.5 & 77.8 \\
6 & 0.77 & 4 & 78.9 & 80.3 \\
7 & 2.01 & 4 & 79.3 & 78.5 \\
8 & 1.81 & 2 & 80.9 & 79.5 \\
9 & 2.79 & 2 & 80.1 & 78.2 \\
10 & 3.47 & 2 & 83.8 & 84.5 \\
11 & 4.26 & 1.5 & 85.4 & 86.0 \\
12 & 3.49 & 1.5 & 88.0 & 89.0 \\
13 & 12.0 & 1.5 & 86.4 & 86.1 \\
14 & 7.33 & 1 & 95.4 & 95.0 \\
15 & 12.49 & 1 & 90.4 & 90.2 \\
\hline
\end{tabular}


The adequacy of the proposed model was determined according to the determination coefficient (R2), F-value and p-value (see Table 4). An R2 of at least 0.80 is indicative of the good fit of a model (Dahunsi et al., 2016). The F-value is based on the comparison between the variance related with all terms and the residual variance; whereas, the p-value refers to the probability value which is related to the F-value for all terms (Dahunsi et al., 2016). The model R2, F-value and P-value of $0.9638,47.93$ and $<0.0001$, respectively, obtained for this optimization study indicated that the model was suitable to predict the COD removal efficiency. The adjusted determination coefficient (R2 Adj) and predicted determination coefficient (R2 Pred) values obtained were 0.9437 and 0.9097, respectively. The R2 values are similar to those reported in literature for the optimization process using RSM for wastewater treatment (Bustillo-Lecompte \& Mehrvar, 2017). The significance of the individual variables and their interactions in the model were determined by $p$ values less than 0.05. According to Table 4, the A2 and B2 had an insignificant effect on the COD removal efficiency. Therefore, Eq. 2 was reduced to Eq. 3, which represents the final quadratic model equation used to estimate the COD removal efficiency. Eq. 2 correlates the experimental results and the predicted results and is thus considered as a good fit.

COD Removal efficiency $=121.64+51.38 \mathrm{~A}+42.57 \mathrm{~B}+44.44 \mathrm{AB}$

Figure 2 illustrates the 3-D response surface plot representing the effect of the OLR and HRT on the COD removal efficiency achieved by the SGBR during the treatment of PSW. It is evident that the SGBR efficiency is affected by both the organic and hydraulic loading rates for the reactor. Thus, a balance between the two operating conditions must be maintained in order to ensure the stable and efficient operation of the SGBR as well as to prevent reactor failure. In other words, in order to allow the micro-organisms sufficient time for the biodegradation of the organic matter (i.e. COD) present in the PSW, certain conditions must not be exceeded so as to prevent overfeeding of the micro-organisms (Nayona, 2010). The optimal SGBR performance with regard to the maximum COD removal efficiency was predicted for an OLR of $12.49 \mathrm{~g} \mathrm{COD/Lday} \mathrm{and} \mathrm{a} \mathrm{HRT} \mathrm{of} 1$ day (24 h), resulting in a 95.5\% COD removal efficiency. Similarly, Muhamad et al. (2013) reported a COD removal efficiency $>90 \%$ at an HRT of 1 day $(24 \mathrm{~h})$ for the optimization of the COD removal of a granular activated carbon sequencing batch biofilm reactor (GAC-SBBR) treating recycled paper wastewater. Ideally, it is desired that the SGBR be operated at a maximum OLR, for the shortest HRT, in order to achieve the highest COD removal efficiency without adversely impacting the SGBR performance and operation for high throughput rates of treated PSW. However, since the SGBR is a biological system there might be other factors other than the organic and hydraulic loading, which might affect the SGBR performance such as the activity of the methanogens (Oh et al., 2015); however, these were not identified and investigated in this study. Figure 2 illustrates the 3-D response surface plot representing the effect of the OLR and HRT on the COD removal efficiency achieved by the SGBR during the treatment of PSW. It is evident that the SGBR efficiency is affected by both the organic and hydraulic loading rates for the reactor. Thus, a balance between the two operating conditions must be maintained in order to ensure the stable and efficient operation of the SGBR as well as to prevent reactor failure. In other words, in order to allow the micro-organisms sufficient time for the biodegradation of the organic matter (i.e. COD) present in the PSW, certain conditions must not be exceeded so as to prevent overfeeding of the microorganisms (Nayona, 2010). The optimal SGBR performance with regard to the maximum COD removal efficiency was predicted for an OLR of $12.49 \mathrm{~g} \mathrm{COD/Lday} \mathrm{and} \mathrm{a} \mathrm{HRT} \mathrm{of} 1$ day $(24 \mathrm{~h})$, resulting in a 95.5\% COD removal efficiency. Similarly, Muhamad et al. (2013) reported a COD removal efficiency $>90 \%$ at an HRT of 1 day $(24 \mathrm{~h})$ for the optimization of the COD removal of a granular activated carbon sequencing batch biofilm reactor (GAC-SBBR) treating recycled paper wastewater. Ideally, it is desired that the SGBR be operated at a maximum OLR, for the shortest HRT, in order to achieve the highest COD removal efficiency without adversely impacting the 
SGBR performance and operation for high throughput rates of treated PSW. However, since the SGBR is a biological system there might be other factors other than the organic and hydraulic loading, which might affect the SGBR performance such as the activity of the methanogens (Oh et al., 2015); however, these were not identified and investigated in this study.

Table 4 Analysis of variance (ANOVA) of the quadratic model for chemical oxygen demand (COD) removal efficiency

\begin{tabular}{|c|c|c|c|c|c|}
\hline Source & Sum of squares & $\begin{array}{ll}\text { Degree } \\
\text { freedom }\end{array}$ & Mean square & F-value & $\begin{array}{l}\text { p-value } \\
\text { Prob }>\text { F }\end{array}$ \\
\hline Model & 818.36 & 5 & 163.67 & 47.93 & $<0.0001$ \\
\hline $\mathbf{A}$ & 75.90 & 1 & 75.90 & 22.22 & 0.0011 \\
\hline B & 54.42 & 1 & 54.42 & 15.93 & 0.0031 \\
\hline $\mathbf{A B}$ & 34.03 & 1 & 34.03 & 9.96 & 0.0116 \\
\hline $\mathbf{A}^{2}$ & 0.058 & 1 & 0.058 & 0.017 & 0.8995 \\
\hline $\mathbf{B}^{2}$ & 7.78 & 1 & 7.78 & 2.28 & 0.1656 \\
\hline Residual & 30.74 & 9 & 3.42 & & \\
\hline $\mathbf{R}^{2}$ & 0.9638 & $\mathbf{R}^{2}$ Adj & 0.9437 & $\mathbf{R}^{2}$ Pred & 0.9097 \\
\hline
\end{tabular}

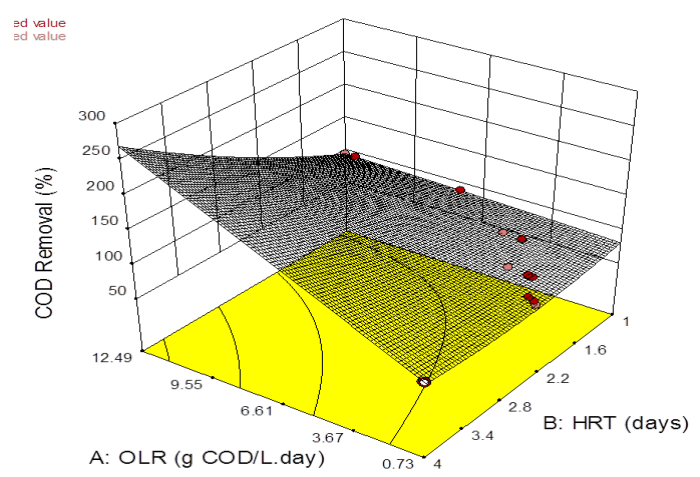

Figure 2 3-D response surface for the COD removal efficiency as a function of variables A: OLR and B: HRT

\section{CONCLUSIONS}

The SGBR was able to consistently reduce the organic matter content of the pre-filtered PSW throughout its 138 days of operation. The SGBR efficiently reduced the COD by $80 \%$ on average for HRTs ranging from 24 to $96 \mathrm{~h}$ and average OLRs of between 0.78 and $5.74 \mathrm{~g} \mathrm{COD/Lday.}$ Neither an increase nor decrease in the HRT or OLR had an adverse effect on the treatment efficiency of the SGBR in terms of its biological performance. Furthermore, the SGBR successfully treated the PSW to within the industrial effluent municipal discharge limits with regard to the COD. Despite the effluent COD averaging at $3649 \mathrm{mg} / \mathrm{L}$, which is below the permitted discharge limit of $5000 \mathrm{mg} / \mathrm{L}$, further reduction of COD to a value below $1000 \mathrm{mg} / \mathrm{L}$ would eliminate penalties imposed on industries. Using RSM, the optimal SGBR performance with regard to the maximum COD removal efficiency was predicted for an OLR of $12.49 \mathrm{~g} \mathrm{COD/Lday} \mathrm{and} \mathrm{a} \mathrm{HRT} \mathrm{of} 24 \mathrm{~h}$ (1 day), resulting in a $95.5 \%$ COD removal efficiency. The model $\mathrm{R}^{2}$ of 0.9638 indicated that the model is a good fit to predict the COD removal efficiency for the SGBR. Further experiments should be done to quantitatively and qualitatively evaluate the biogas produced by the SGBR treating PSW; and the data obtained may be used to support the experimental and RSM data.

\section{REFERENCES}

Basitere, M., Rinquest, Z., Njoya, M., Sheldon, M.S. \& Ntwampe, S.K.O. 2017. Treatment of 
poultry slaughterhouse wastewater using a static granular bed reactor (SGBR) coupled with ultrafiltration (UF) membrane system. Water Science \& Technology, 76(3):106-114.

Bustillo-Lecompte, C.F. \& Mehrvar, M. 2017. Treatment of actual slaughterhouse wastewater by combined anaerobic-aerobic processes for biogas generation and removal of organics and nutrients: An optimization study towards a cleaner production in the meat processing industry. Journal of Cleaner Production, 141:278-289.

Chong, S., Sen, T.K., Kayaalp, A. \& Ang, H.M. 2012. The performance enhancements of upflow anaerobic sludge blanket (UASB) reactors for domestic sludge treatment - A State-of-the-art review. Water Research, 46:3434-3470.

Dahunsi, S.O., Oranusi, S., Owolabi, J.B. \& Efeovbokhan, V.E. 2016. Mesophilic anaerobic codigestion of poultry dropping and Carica papaya peels: Modelling and process parameter optimization study. Bioresource Technology, 216:857-600.

Debik, E. \& Coskun, T. 2009. Use of the Static Granular Bed Reactor (SGBR) with anaerobic sludge to treat poultry slaughterhouse wastewater and kinetic modeling. Bioresource Technology, 100:2777-2782.

Del Nery, V., De Nardi, I.R., Damianovic, M.H.R.Z., Pozzi, E., Amorim, A.K.B. \& Zaiat, M. 2007. Long-term operating performance of a poultry slaughterhouse wastewater treatment plant. Resources, Conservation and Recycling, 50:102-114.

Ellis, T. G. \& Evans, K. M. 2008. A new high rate anaerobic technology, the static granular bed reactor (SGBR), for renewable energy production from medium strength waste streams. Waste Management and the Environment, IV:141-150.

Evans, E.A. 2004. Competitive evaluation and performance characterization of the Static Granular Bed Reactor. Ph.D. dissertation. Iowa State University.

Lim, S.J. 2008. Swine wastewater treatment by the static granular bed reactor. MSc. thesis. Iowa State University.

Lim, S.J. \& Fox, P. 2011. Evaluation of a static granular bed reactor using a chemical oxygen demand balance and mathematical modelling. Bioresource Technology, 102:6399-6404.

Mach. K. 2004. Fundamentals of the static granular bed reactor. Ph.D. dissertation. Iowa State University.

Muhamad, M.H., Abdullah, S.R.S., Mohamed, A.B., Rahman, R.A. \& Khadum, A.A.H. 2013. Application of response surface methodology (RSM) for optimization of COD, $\mathrm{NH}_{3}-\mathrm{N}$ AND 2,4DCP removal from recycled paper wastewater in pilot-scale granular activated carbon sequencing batch biofilm reactor (GAC-SBBR), Journal of Environmental Management, 121:179-190.

Mohamed, A.G. 2014. Investigation of performance of a submerged anaerobic membrane bioreactor (ANMBR) treating meat processing wastewater. M.Sc. thesis. University of Waterloo.

Nayona, S.E. 2010. Anaerobic digestion of organic solid waste for energy production. Germany: KIT Scientific Publishing.

Oh, J. H. 2012. Performance evaluation of the pilot-scale static granular bed reactor (SGBR) for industrial wastewater treatment and biofilter treating septic tank effluent using recycled rubber particles. Ph.D. thesis. Iowa State University.

Oh, J.H., Park, J. \& Ellis, T.G. 2015. Performance of on-site pilot static granular bed reactor (SGBR) for treating dairy processing wastewater and chemical oxygen balance modeling under different operational conditions. Bioprocess and Biosystem Engineering, 38:353-363.

Park, J., Oh, J. H., Evans, E. A, Lally, M. F. Hobson, K. L. \& Ellis, T. G. 2012a. Industrial wastewater treatment by on-site pilot static granular bed reactor (SGBR). Water Practice \& Technology, 7(1):1-11.

Wellinger, A., Murphy, J. \& Baxter, D. 2013. The Biogas Handbook: Science, Production and Applications. Oxford: Woodhead Publishing. 